\title{
Transatlantica
}

Revue d'études américaines. American Studies Journal

\section{Réponse à Mme DESAFY-GRIGNARD au sujet de son ouvrage Arthur Miller : une vie à l'œuvre}

\section{Trudy Bolter}

\section{Q OpenEdition}

1 Journals

\section{Édition électronique}

URL : http://journals.openedition.org/transatlantica/1158

DOI : 10.4000/transatlantica.1158

ISSN : 1765-2766

Éditeur

AFEA

\section{Référence électronique}

Trudy Bolter, « Réponse à Mme DESAFY-GRIGNARD au sujet de son ouvrage Arthur Miller : une vie à l'œuvre », Transatlantica [En ligne], 1 | 2003, mis en ligne le 16 novembre 2006, consulté le 29 avril 2021. URL : http://journals.openedition.org/transatlantica/1158; DOI : https://doi.org/10.4000/ transatlantica. 1158

\section{Ce document a été généré automatiquement le 29 avril 2021.}

\section{c) (i)}

Transatlantica - Revue d'études américaines est mis à disposition selon les termes de la licence Creative Commons Attribution - Pas d'Utilisation Commerciale - Pas de Modification 4.0 International. 


\title{
Réponse à Mme DESAFY-GRIGNARD au sujet de son ouvrage Arthur Miller: une vie à l'œuvre
}

\author{
Trudy Bolter
}

\section{NOTE DE L'ÉDITEUR}

Cette réponse fait suite à un droit de réponse au compte rendu par Trudy Bolter (Institut d'Etudes Politiques, Université Montesquieu-Bordeaux IV) de Christine DESAFY-GRIGNARD. Arthur Miller : Une vie à l'œuvre. Paris : Michel Houdiard Editeur, 2003. Ce texte clôt l'échange entre les deux intéressées et le public selon les règles de la déontologie de l'édition.

\begin{tabular}{llllll} 
RÉPONSE & \multicolumn{2}{c}{ à } & Mme & \multicolumn{3}{c}{ DESAFY-GRIGNARD } \\
au sujet de son ouvrage & Arthur Miller : une vie à l'oeuvre
\end{tabular}

Un compte rendu n'est pas forcément un résumé neutre : rien ne m'oblige à répéter en l'étoffant la quatrième page de couverture de l'ouvrage. « Lu par ... » dit bien qu'il s'agit d'une vision personnelle. J'avoue que j'ai lu ce livre avec surprise, une émotion très vive, pas toujours très positive. Je conteste certaines approches, qui m'apparaissent approximatives, pas assez complètes, quelques tournures qui semblent, à mon avis, ne pas vraiment correspondre à la pensée de l'auteur telle qu'elle la présente dans son droit de réponse.

2 La haine revient partout. Elle se nourrit du stéréotype, de l'analyse rapide, du raccourci. Dans ces conditions, utiliser un langage neuf, précis, devient à mes yeux un devoir professionnel, voire citoyen. Je ne mets pas en cause dans ce domaine le cœur de la pensée, les opinions profondes de Mme. Desafy-Grignard, et je ne souhaite donc en aucun cas en faire un débat personnel. Mais j'ai remarqué dans son texte la présence 
occasionnelle de certains stéréotypes, qu'il conviendrait d'aborder avec plus de distance critique, surtout pour une auteure sensible, convaincue de la valeur des individus aussi bien que des tendances plus générales des cultures juives qu'elle étudie depuis longtemps, semble-t-il. Ces clichés sont certainement « loin de ses propos »elle a raison de protester. Mais en toute honnêteté je ne pourrais jamais dire, pour la satisfaire, qu'ils n'y sont pas présents.

3 Soyons clairs : je ne me suis jamais méprise sur son respect de la judéité, Mais c'est justement pour cela que j'ai pensé que son ouvrage était inachevé, trop rapidement rédigé : le temps donne du recul,, le texte qui repose se décante et se clarifie. J'ai trop d'expérience de l'écriture pour sous-estimer le nombre de malentendus que peut susciter la plume trop rapide d'un homme ou d'une femme de bonne volonté. Je récuse non les convictions de l'auteure, mais les glissements de langage qui entachent parfois les pages écrites par Mme Desafy-Grignard - et elle n'est pas la seule - ; c'est justement pour cela que je réagis avec tant d'énergie.

4 Je suis une lectrice un peu particulière, c'est vrai, car j'ai rédigé une thèse comparatiste qui traite, entre autres, d'Eugène Ionesco. Le point de vue de Ionesco, ajouté à mon scepticisme naturel, crée des difficultés, dont je suis, peut-être de manière perverse, assez fière, car je pense que la résistance aux stéréotypes est une forme utile de rébellion, de résistance au sens plus large. Reparlons un peu de la « yiddish mamma » : je n'ai jamais pu comprendre en quoi elle diffère de la mamma italienne. Quant au terme de " juif psychologique », personnage postulé par Yosef Yerushalmi que cite Madame Desafy-Grignard, je l'accepte mal. Pour moi, il s'agit d'un " stéréotype distingué », pour paraphraser ce cher Ionesco. Existe-t-il ? Affirmer n'est pas prouver. Où sont les exemples, les exceptions? J'aime mieux parler de certains juifs, que d'un Juif de partout et de toujours, doté de "traits de caractère inaliénables ». Peut-on approuver de parler ainsi de catholiques, d'Américains, de Français , et j'en passe?

5 L'analyse de Sievers, que l'auteur développe peu, est tout simplement étonnante. Quelle est cette logique qui conduit un auteur à " trahir (quel verbe !) sa judéité » " par ses intuitions sur Freud ». Qui se rassemble s'assemble ? Et Proust, alors ? Lawrence ? Shakespeare?

6 Je veux redire ici (fidèle à Ionesco) que le stéréotype (verbal ou intellectuel) se prête à la dérive. Il faut l'éviter, et apprendre à nos étudiants à s'en méfier. Et ce, dans tous les domaines. If not now, then when ? If not us, then who?

7 Il y lieu de présenter en guise de complément à la réponse ci-dessus une partie, un peu remaniée pour les besoins de la cause, du long article que m'a inspiré dans un premier temps cette lecture. Je l'ai coupé pour me conformer à la longueur standard des comptes rendus de Transatlantica, mais la version courte était peut-être trop condensée. Je reprends donc ci-dessous en détail certains des arguments que j'ai inclus dans mon compte rendu, en me référant à des extraits plus extensifs de l'ouvrage concerné.

8 Dans son droit de réponse, Mme Desafy-Grignard souligne son approche " socioculturelle " de l'œuvre de Miller. Mais au fil du texte, on remarque quelques approximations sur le plan de l'histoire culturelle,.qui donnent au lecteur (un étudiant ?) un tableau tellement simplifié qu'il en vient à être erroné. Voici par exemple la première partie du texte de la note 7, qui se référe à la page 287, et apparaît page 419 : 
elle est destinée à expliciter l'affirmation par Miller de son ignorance de l'œuvre de Brecht à l'époque du Commis-voyageur:

En 1950, les producteurs de Broadway n'auraient pas songé à mettre en scène les pièces du Théâtre épique de et du Théâtre de l'Absurde de Ionesco qui sont déjà joués en Europe.

Cette note est rajoutée comme information complémentaire censée élargir la culture du lecteur, étoffer le contexte de l'affirmation de Miller. La première partie de cette affirmation brève n'est pas erronée, mais l'ensemble paraît hâtif et incomplet si l'on se reporte au détail de la chronologie de l'histoire du théâtre international, car il s'avère qu'en 1950, le théâtre européen lui-même venait tout juste de songer à produire les œuvres dramatiques de Ionesco (que lui-même venait tout juste - en 1949 - de se mettre à les écrire): la première de la Cantatrice chauve eut lieu le 16 mai 1950 au Théâtre des Noctambules. Quant à Brecht, les productions classiques du théâtre épique - essentiellement les productions des grandes pièces de Brecht écrites pendant les années de son errance (1933-1941) - ont été élaborées avec sa femme Hélène Weigel, au Berliner Ensemble fondé en 1949, et installé dans son propre théâtre en 1954, deux ans avant sa mort. Bien que les pièces de Brecht n'aient pas, au début, trouvé en Amérique la réussite espérée (c'était aussi le cas de ses scénarios hollywoodiens, à l'exception de Hangmen Also Die /Les bourreaux meurent aussi, Fritz Lang, 1943) certaines furent bel et bien produites en Amérique dès 1933 - rarement, c'est vrai. L'ignorance de Brecht qu'affiche Miller dans Timebends est un peu surprenante quand on sait que le 7 décembre 1947, le Galileo de Brecht fut produit à New York, dans une traduction de Charles Laughton, qui jouait le rôle titre, et une mise en scène de Joseph Losey - 6 représentations seulement, malheureusement. Mais elles ont existé.

10 Pour revenir à Broadway, si Ionesco dut attendre 1961 pour y être joué, et Brecht 1963, L'Opéra de Quat'sous de Brecht et Kurt Weill tint sept ans sur Off-Broadway à partir de 1954 - attirant des centaines de milliers de spectateurs, et faisant largement connaître le nom de l'auteur. (The Bald Soprano se joua off-Broadway en 1958). La théorie du v-effekt, amorcée plus tôt, est pleinement développée par Brecht dans son Petit Organon du Théâtre qui date de 1949. ( L'influence de Brecht alla croissant à partir de la production de Mère Courage qu'il mit en scène en 1949, année qui apparaît comme une sorte d'annus mirabilis du théatre occidental). Cette toile de fond très riche méritait, à mon avis, un traitement plus ample, mais les choix d'un auteur sont bien évidemment question de goût, je l'accorde.

11 Le style est parfois imprécis. En parlant du chef d'œuvre de Miller, Mort d'un commis-voyageur, l'une des œuvres emblématiques de la littérature américaine du XXe siècle, et peut-être de tous les temps, l'auteur nous dit :

Le dramaturge a écrit sa pièce au début des années 50 qui demeurent la période du plus grand boom économique de l'histoire mondiale. Or la vie de Willy Loman, l'échec de son fils Biff, sont à la fois l'illustration et la négation de la vague d'optimisme et de matérialisme qu'est censée soulever cette croissance économique soudaine chez beaucoup d'Américains. Voilà pourquoi elle est et demeure une pièce populaire mais contestée. (133)

Comment comprendre le paragraphe cité? Mort d'un commis voyageur a été créée sur Broadway en 1949. Certes, la pièce a été produite au seuil des années cinquante, à la veille de cette décennie : mais si rétrospectivement, en 2003, date du livre de Mme Desafy-Grignard, la pièce nous apparaît comme " l'illustration et la négation de la vague d'optimisme ", comment, pouvait-elle produire cet effet sur le spectateur de 
l'époque, avant même que cette période se soit déroulée ? C'est donc l'expérience de la vie américaine postérieure à l'écriture de la pièce qui lui donne rétroactivement son sens ? Comment dans ce cas expliquer la réception exceptionnelle (spectateurs en pleurs, trop émus pour applaudir), qui l'accueillit en 1949, avant même que démarrent les années cinquante?

Un peu plus loin sur la même page, est abordée la question épineuse, qui a fait couler beaucoup d'encre et inspiré plus d'un cours de littérature, du caractère tragique (ou non) de Mort d'un commis voyageur:

Dans les siècles passés on parlait de la mort d'un archevêque, d'un roi, à l'époque moderne d'un président, parce que ce sont des gens importants, mais un commis voyageur qui de plus, est laid, banal, impécunieux et vieux !'Voilà un titre qui détruit plus d'un mythe, qui dérange plus les mentalités dans un pays où beauté, jeunesse, argent, réussite sont des valeurs essentielles. (134)

14 Cette discussion très courte ne comporte pas d'exemples. Se réfère-t-on à Murder in theCathedral (1935), d'Eliot ? Connaît-on des exemples de tragédie portant sur un président ? Ce traitement rapide banalise et schématise à la fois la question de l'essence tragique, et celle des valeurs américaines, réduites ici à quelques clichés sommaires qui relèvent plus de la vulgarisation journalistique que de l'analyse .

Ce recours au raccourci, au nivellement et au stéréotype, me semble encore plus regrettable dans le domaine de la judéité de Miller lui-même. Effectivement, Mme Desafy-Grignard s'attache à montrer que ce "juif laïcisé ", issu d'une famille peu pratiquante, époux de trois chrétiennes laïcisées, est un auteur " juif », et, en plus, d'une certaine façon, religieux. (381)

Vie et œuvre attestent que Miller possède une personnalité riche et pleine de contradictions, fruit d'un double héritage culturel ; la culture protestante, plus particulièrement puritaine, des pays anglo-saxons et le patrimoine ethnique juif. La culture protestante, c'est l'homme " extérieur ", tourné vers l'action, les autres, et l'avenir. Le patrimoine juif, c'est l'homme " intérieur » enclin à l'introspection, tiraillé par des sentiments ambivalents et tourné vers le passé. (380)

16 Le point de vue de l'auteur se défend, et se rapproche d'ailleurs de celui d'Harold Bloom. Mais comment définit-on le « juif » ? Dans l'extrait ci-dessus, le double portrait du protestant-juif me semble schématique, déterministe, les étiquettes sont avancées avec trop de confiance, trop peu de nuances. La culture " puritaine ", la culture ethnique " juive ", sont-elles des blocs tellement distincts, aussi monolithiques, - et somme toute réfractaires l'un à l'autre - éternellement insolubles malgré l'effervescence bouillonnante de la culture américaine qui se re-brasse sans cesse ? Je ne partage pas sur ce sujet l'opinion de Christine Desafy-Grignard, mais son point de vue pourrait mieux se défendre si les stéréotypes qu'elle emploie avaient été mis en question. Or, ils font souvent figure de « sagesse populaire » tout particulièrement dans la première partie du livre qui s'intitule " la judéité ». On peut citer pour exemple les passages ci-dessous :

(17) Sa mère, Augusta, est plus superstitieuse (un trait de caractère chez les femmes juives que l'on retrouve chez plusieurs des hérö̈nes de l'œuvre) que pieuse, plus attirée par le côté occulte de sa religion que par l'orthodoxie du dogme

(19) À la double culture judaïque et germanique acquise pendant la première enfance, Isidore (le père d'Arthur Miller) dût en ajouter une troisième, la culture américaine : il l'assimila pleinement, si l'on en juge par son désir constant d'évolution, ce devenir perpétuel qui est un trait du caractère juif ; l'errance 
imposée à travers les siècles, source de souffrance, a développé chez le Juif la qualité du caméléon : une grande adaptabilité.

(21) Sans pouvoir attribuer avec certitude les traits de caractère d'Esther Simon dans Pas si méchant que ça à l'une ou l'autre des sœurs d'Augusta, il est à peu près sûr que Miller s'est inspiré de leur caractère pour ce personnage. Ce dernier possède en effet plusieurs traits caricaturaux de l'épouse et de la mère juives telles qu'on les montre dans les pièces de boulevard

Reproduire les stéréotypes quels qu'ils soient a l'effet de les perpétuer, les renforcer. Le fait même de les utiliser nous rend vulnérables aux effets pervers qui sont parfois inscrits de façon indélébile dans ces figures. Un exemple : le juif « caméléon », victime de diaspora ou génocide, displaced person par excellence : c'est une figure hautement symbolique, à résonance universelle. Mais ce terme peut susciter plus d'une interprétation malveillante.

Il est pour moi absolument clair que Mme Desafy-Grignard respecte - vénère même Miller, sa judéité, la judéité en général, et je ne mets pas en cause le fond de ses propos, son respect de la différence, que je suppose entier. Son livre ne cherche qu'à illustrer la démarche de l'auteur américain, beau sujet s'il en était. Mais le langage ne doit pas stagner, la " véracité » du cliché doit être constamment testée pour savoir si sa couleur n'a pas viré à l'équivoque. Aborder un texte littéraire par le biais de l'ethnicité est une démarche risquée, parfois réductrice, qui tend à conforter, si l'on n'y prête garde, les préjugés dans lesquels elle peut s'enfermer, au détriment du questionnement qui demeure le fondement de l'entreprise critique, et - selon moi - de la vie.

INDEX

Thèmes : Recensions 